\title{
Renal interstitial fibrosis: Remembrance of things past?
}

Commentary

See related article, pages $341-350$.

\author{
Doris Herzlinger
}

Department of Physiology and Biophysics and Department of Urology, Weill Medical College of Cornell University, 1300 York Avenue, Room D501, New York, New York 10021, USA. Phone: (212) 746-6377; Fax: (212) 746-8690; E-mail: daherzli@med.cornell.edu.

J. Clin. Invest. 110:305-306 (2002). doi:10.1172/JCI200216377.

The decline in renal function seen in a variety of pathological states is associated with an increase in the number and changes in the protein synthetic activity of tubular interstitial cells. However, the relationship between expansion of the interstitial compartment (interstitial fibrosis) and loss of renal epithelial tubules and capillaries remains unclear. Interstitial pathologies accompanying renal failure may merely be coincident with tubular degeneration. Alternatively, interstitial pathologies may cause tubular degeneration. Finally, it is possible that "pathological" changes in the interstitial compartment reflect the kidney's attempts to repair damaged tubules. Discriminating among these possibilities has been difficult, in part because the function of interstitial cells in the healthy kidney remains poorly understood. A new study by Iwano et al. in this issue of the JCI (1) suggests that recent advances in the field of kidney development may provide insight into this important clinical problem.

\section{Interstitial cells in early renal epithelial differentiation}

The mammalian kidney derives from two embryonic tissues: the ureteric bud, which forms the renal collecting system, and the metanephric mesenchyme, which differentiates into nephrons and tubular interstitial cells (2). Inductive interactions between these two tissues regulate kidney morphogenesis (3). The metanephric mesenchyme supports the viability, growth, and branching of the ureteric bud as it forms the renal collecting system. In turn, the growth of the ureteric bud or nascent collecting system induces multipotent nephron progenitors of the metanephric mesenchyme to differentiate into glomerular and tubular epithelial cell types $(3,4)$. Several of the signaling molecules, receptors, and transcrip- tion factors required for these inductive interactions have been identified [see ref. 5 for review].

Although inductive interactions between mesenchymal nephron progenitors and the ureteric bud are essential for kidney morphogenesis, they are not sufficient. We discovered that functional tubular interstitial cells are also essential for renal epithelial morphogenesis (6). Targeted deletion of brain factor 2 (BF-2), a transcription factor expressed exclusively by interstitial cell progenitors in the metanephric blastema, severely perturbs nephron differentiation and

\section{Recruitment of these cells to the interstitium is the kidney's attempt to restore an embryonic environment in which tubular interstitial cells could support repair of injured tubules if the obstruction were relieved.}

collecting system morphogenesis in vivo. Abnormal renal epithelial phenotypes have also been observed in mice lacking functional expression of other transcription factors that are expressed by interstitial cells in the developing kidney. Collectively, these studies provide direct evidence that renal interstitial cells are required for renal epithelial morphogenesis (6-9).

\section{Phenotypic conversion of tubular epithelia to interstitial cells following ureteral obstruction}

The study by Iwano et al. (1) suggests that the mesenchymal-to-epithelial conversion that occurs during embryonic development reverses in response to ureteral obstruction. Working with transgenic mice engineered to express the marker LacZ in tubular epithelia, Iwano et al. imposed a unilateral ureteral obstruction to injure one of each animal's kidneys. By following LacZ staining, the authors could unambiguously identify tubular epithelia and cells deriving from them. They show here that LacZ-expressing epithelia assume an abnormal morphology, become disorganized, and move into the interstitium. Moreover, these interstitial cells derived from epithelia upregulate the expression of fibroblast-specific protein-1 (FSP1), a cytoskeleton-binding protein that is normally expressed in fibroblasts but not epithelia (10). In control kidneys, as expected, LacZ expression is restricted to tubular epithelia, whereas FSP1 localizes to interstitial fibroblasts. Thus, although the proximate signals that induce obstructive injury remain unclear, the present data provide strong support for the hypothesis that obstruction causes tubular cells to undergo an epithelialto-mesenchymal conversion.

Another crucial question resolved in this study is whether $\mathrm{FSP}^{+}$cells derived from tubular epithelia play a significant role in fibrogenesis, specifically the deposition of abnormal amounts and types of collagens. The observed epithelial-to-mesenchymal conversion might represent a consequence, not a cause, of fibrogenesis. Iwano et al. address this alternative in an indirect but convincing manner by staining the injured and control tissue for HSP47, a stress-induced, collagenspecific chaperone protein that is presumed to serve as a marker of cells depositing abnormal amounts and types of collagens into the renal interstitium. Results of these experiments demonstrate that HSP47 expression is induced in mesenchymal cells deriving from tubular epithelia and support the hypothesis that the mesenchymal- 
to-epithelial conversion documented plays a direct role in fibrogenesis.

\section{Restoration of the embryonic environment in the injured kidney}

Collectively, these results, along with other experiments showing that bone marrow-derived cells home to the interstitial compartment of the obstructed kidney, indicate that cells derived from a variety of sources are recruited to the renal interstitium in response to obstructive injury. Perhaps recruitment of these cells to the interstitium is the kidney's attempt to restore an embryonic environment in which tubular interstitial cells could support repair of injured tubules if the obstruction were relieved. It may also be the case, however, that this response is maladaptive and exacerbates the primary injury caused by the obstruction. Thus, the Iwano study also raises the possibility that novel therapies to prevent or slow the progression of end-stage renal disease associated with obstruction could target the molecular events that induce renal epithelia to revert to a mesenchymal phenotype.

1. Iwano, M., et al. 2002. Evidence that fibroblasts derive from epithelium during tissue fibrosis. J. Clin. Invest. 110:341-350. doi:10.1172/JCI200215518.

2. Saxén, L. 1987. Organogenesis of the kidney. Cambridge University Press. Cambridge, United Kingdom. 173 pp

3. Grobstein, C. 1957. Trans-filter induction of tubules in mouse metanephrogenic mesenchyme. Exp. Cell Res. 10:424-440.
4. Herzlinger, D., et al. 1992. Metanephric mesenchyme contains multipotent stem cells whose fate is restricted after induction. Development. 114:565-572.

5. Schedl, A., and Hastie, N.D. 2000. Cross-talk in kidney development. Curr. Opin. Genet. Dev. 10:543-549.

6. Hatini, V., et al. 1996. Essential role of stromal mesenchyme in kidney morphogenesis revealed by targeted disruption of Winged Helix transcription factor BF-2. Genes Dev. 10:1467-1478.

7. Mendelsohn, C., et al. 1999. Stromal cells mediate retinoid-dependent functions essential for renal development. Development. 126:1139-1148.

8. Batourina, E., et al. 2001. Vitamin A controls epithelial/mesenchymal interactions through Ret expression. Nat. Genet. 27:74-78.

9. Quaggin, S.E., et al. 1999. The basic-helix-loophelix protein pod 1 is critically important for kidney and lung organogenesis. Development. 126:5771-5783.

10. Strutz, F., et al. 1995. Identification and charac terization of a fibroblast marker: FSP1. J. Cell Biol. 130:393-405. 